NASA Technical Memorandum 103689

AIAA-91-0043

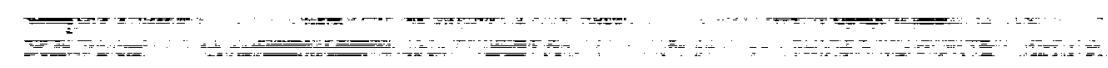 \\ A Steadying Effect of Acoustic Excitation on Transitory Stall
}

K.B.M.Q. Zaman

Lewis Research Center

Cleveland, Ohio

Prepared for the

29th Aerospace Sciences Meeting

sponsored by the American Institute of Aeronautics and Astronautics

Reno, Nevada, January 7-10, 1991

\section{N/SN}

(NASA-TM-1036BY) A STEAOYING EFFECT OF

$N 91-13420$ ACOUSTIC EXCITATIAN ON TRANSITOPY STALL (NASA) $17 \mathrm{p}$

CSCL DIA 


\title{
A STEADYING EFFECT OF AOOUSTIC EXCITATION \\ ON TRANSITORY STALL
}

by

\author{
K. B. M. Q. Zaman \\ National Aeronautics and Space Administration \\ Lewis Research Center \\ Cleveland, OH 44135
}

\section{Abstract:}

The effect of acoustic excitation on a class of separated flows with a transitional boundary layer at the point of separation is considered in this paper. Experimental results on the flow over airfoils, a two-dimensional backward-facing step, and through large angle conical diffusers are presented. In all cases, the separated flow undergoes large amplitude fluctuations, much of the energy being concentrated at unusually low frequencies. In each case, an appropriate high frequency acoustic excitation is found to be effective in reducing the fluctuations substantially. The effective excitation frequency scales on the initial boundary layer thickness and the effect is apparently achieved through 'acoustic tripping' of the separating boundary layer.

\section{Introduction:}

Wall bounded separated flows often exhibit fluctuations at unusually low frequencies. 1-5 The frequency is unusually low in the sense that it is order(s) of magnitude lower than what is expected from the stability characteristics of the upstream boundary layer, the separated shear layer and even of the downstream wake e.g. in the case of flow over an airfoil. 5 The flow fluctuations are of large amplitudes and are often accompanied by large unsteady forces. Here, the term transitory stall will be used in a broad sense to refer to such low frequency, large amplitude flow fluctuations in stalled flows. Transitory stall have been observed even with a fully turbulent state of the separating boundary layer 3,4 . However, in this paper we restrict ourselves to cases where the separating boundary layer is transi- 
tional. It is in this type of flows where acoustic excitation can have a very pronounced effect in reducing the unsteady fluctuations.

Examples of the steadying effect for the flow over airfoils were reported in refs. 5,6. Two sets of data are reproduced from ref. 6 in figs. 1 (a) and (b); each shows wake velocity spectra measured about one chord downstream of the trailing edge with and without acoustic excitation. Here, $S t_{S}=f a \sin a / U_{\infty} ; c$ is the airfoil chord, a the angle of attack and $U_{\infty}$ the freestream mean velocity. The excitation frequency in figs. I(a) and (b) correspond to a strouhal number $\left(S t_{S}\right)$ of 18.7 and 1.7 , respectively. The unexcited flows in these cases involved a quasi-periodic switching between stall and unstall. The resulting flow fluctuations were of very large amplitudes and occurred at narrow-band frequencies, the corresponding sts value being an order of magnitude lower than what is expected in the familiar bluff-body shedding. In either of figs. $1(a)$ and (b) it can be seen that acoustic excitation completely suppressed the unsteady oscillation resulting in a remarkably quieter flow.

A similar effect of acoustic excitation was observed in our recent experiments with a backward-facing step and conical diffusers. The primary purpose of the present paper is to document the effect for the various flow configurations, with the hope that common features could be identified which will lead to an understanding of the mechanisms for not only the acoustic excitation effect but also for the low frequency oscillations. As will be shown the suppression by the excitation is a global effect resulting in a flow field that may still be separated but remarkably quieter. Gaining an understanding of the effect is obviously appealing not only from a fundamental fluid dynamics point of view but also in terms of achieving practical control on this class of flows.

Note that there have been previous experiments showing suppression of flow fluctuations under acoustic excitation in free shear flows. 7 A similar effect was also reported for a boundary layer over a flat plate. ${ }^{8}$ But in this paper we focus on the wall-bounded separated flow cases. The bulk of the data presented in the following are from the conical diffuser experiment for which preliminary results were presented in ref. 9.

\section{Experimental facilities:}

The backward-facing step experiment was done in a low speed wind tunnel which has been described in refs. 5,6. A schematic of the test section with 
the model is shown in fig. 2(a). The height of the step (H) is $12.7 \mathrm{~cm}$ and the span to height aspect ratio is six. Data measured at mid-span are presented. Acoustic excitation was imparted by a woofer mounted on the ceiling of the test section; for details see the above references.

The conical diffuser flow facility was described in ref. 9; a schematic of the essential features is reproduced from that reference as fig. 2 (b). The flow, after passing through a large plenum, exited through a contoured nozzle. Each of the straight conical diffusers was attached to a flange at the end of the nozzle. Five diffusers with total diffusion angles $(\psi)$ of $12^{\circ}$, $16^{\circ}, 20^{\circ}, 24^{\circ}$ and $28^{\circ}$ were used in the experiment. The nozzle end and the entrance to the diffusers were machined to provide a smooth junction. A cylindrical section, extending approximately $1.27 \mathrm{~cm}$ on either side of the junction, will be referred to as the 'throat'. The throat was smoothly faired into the beginning of the diffuser section. Also shown in fig. $2(b)$ are the acoustic drivers used for the excitation. A tap at the throat was used to measure the corresponding static pressure $\left(P_{s t}\right)$. Standard hot-wire and pitotstatic measurements were carried out in both the diffuser experiment and in the wind tunnel. Probe traverses and data acquisition were done by automated computer control.

\section{Results and discussion:}

3.1 Flow over backward-facing step: Fig. 3 shows the maximum fluctuation intensity measured at the separation point as a function of the free-stream speed $U_{e} ; U_{e}$ is measured at $x=0$ (see fig. 2a). At each $U_{e}$ the boundary layer was scanned to determine the maximum $u^{\prime}$. At low $U_{e}$, the separating boundary layer is clearly laminar but above $3 \mathrm{~ms}^{-1}$, the fluctuations increase with increasing speed. Around $7 \mathrm{~ms}^{-1}$ the intensity reaches a very high amplitude but subsides with further increase in $\mathrm{U}_{e}$. Measurement at $\mathrm{U}_{e}=12$ $\mathrm{ms}^{-1}$ indicated a nearly fully turbulent state as determined from the mean velocity (U) profile shape factor and the characteristics of the turbulence intensity $\left(u^{\prime}\right)$ distribution in the boundary layer. The higher intensity at relatively lower $U_{e}$ is associated with a transitional boundary layer and is commensurate with several previous experiments on boundary layer transition. A transitioning boundary layer exhibits higher fluctuation intensity, compared to a fully turbulent case, due to intermittent switching between laminar and turbulent states ${ }^{10}$. 
Clearly, the transitional boundary layer reaches a state when the intensity is the highest. This, to be referred to in the following as the 'most highly disturbed' state, occurred in the present flow at $\mathrm{U}_{e} \approx 6.8 \mathrm{~ms}^{-1}$. At this velocity, the boundary layer momentum thickness and shape factor, measured at the separation point, were $1.45 \mathrm{~mm}$ and 2.28 , respectively.

For $\mathrm{U}_{e}=6.8 \mathrm{~ms}^{-1}$, from cursory surveys, an acoustic excitation frequency of $50 \mathrm{~Hz}$ was chosen which resulted in a suppression of the fluctuation intensities downstream. The $\mathrm{U}$ - and $\mathrm{u}^{\prime}$-profiles measured at $\mathrm{x} / \mathrm{H}=0.5$, with and without excitation, are shown in fig. 4. While the U-profile indicates a slight narrowing of the mixing layer under the excitation, the $u^{\prime}$-profile exhibit a significant suppression of the fluctuation intensity especially on the high speed side.

The corresponding $u^{\prime}$-spectra measured at $x / H=0.5$ and $y / H=0.08$ are shown at the top of fig. 5. The spectra for the unexcited flow is clearly characterized by lange amplitude fluctuations at low frequencies. This frequency range is much lower than the characteristic frequency of the large scale coherent structures in the mixing layer at the measurement location. The latter can be estimated from the local momentum thickness ( $3.95 \mathrm{~mm})$ to be about $40 \mathrm{~Hz}$. The origin and morphology of the low frequency flow fluctuations is unclear, important to understand, and is currently under further investigation. Here, let us look at the excitation effect, and clearly, it is the low frequency energy which is reduced the most under the excitation. A similar comment can be made for the $\mathrm{u}^{\prime}$-spectra data at $\mathrm{x} / \mathrm{H}=2$ shown at the bottom of fig. 5. At this location, however, the low frequency fluctuations are significant even under the excitation.

3.2 Flow through conical diffusers: For this flow the boundary layer measurements were done at the throat in the throat Mach number $\left(M_{t}\right)$ range of $0.075-0.4$. These data were reported in ref. 9. The mean velocity profile at the throat was top-hat with a thin boundary layer; throat diameter to momentum thickness ratio was 800 at $M_{t}=0.4$ which dropped to about 400 at $M_{t}$ $=0.1$. Measurements with and without the $16^{\circ}$ diffuser showed no perceptible change in the boundary layer characteristics.

The velocity fluctuation intensity was measured at a fixed location at the diffuser exit plane. The location was chosen to be slightly off-axis so that the contribution from possible helical mode oscillations were also captured. The total r.m.s. intensity normalized by the throat velocity (Ut) for the five diffusers are shown in fig. 6 as a function of $M_{t}$. For the $12^{\circ}$ 
diffuser the flow is attached throughout the $M_{t}$-range covered, resulting in small fluctuations at the exit. With increasing angle of diffusion ( $\psi$ ) increasing separation takes place that manifests in large fluctuations at the exit. It is clear that for a given diffuser the intensity depends on $M_{t}$. In the intermediate range of $M_{t}$ the intensity is lower. At high $M_{t}$, especially with the $16^{\circ}$ diffuser, the intensity is relatively large due to a selfsustaining flow oscillation. 9 This aspect will not be addressed here.

Let us consider the low Mach number range (fig. 6) where very large fluctuations are observed. Note that all diffusers exhibit a similar behavior but the occurence of the peak fluctuations shifts systematically to higher $M_{t}$ with increasing angle of diffusion. These data have symptoms similar to the data for the backward-facing step shown in fig. 3. Consider for example, the curve for the $28^{\circ}$ diffuser case. The throat boundary layer at very low $M_{t}$ is laminar which results in relatively low amplitude fluctuations downstream. Around $\mathrm{M}_{t}=0.2$ the boundary layer is believed to attain the 'most highly disturbed' state which causes the largest amplitude fluctuations. But the intensity subsides at higher $M_{t}$ presumably when the throat boundary layer approaches a fully turbulent state.

The large fluctuation intensities at the lower Mach numbers are also due to energy at unusually low frequencies as can be observed from the r.m.s. velocity spectra shown in fig. 7 for the five diffusers. These data are measured at the exit plane, for the unexcited flow at $M_{t}=0.075$. The trace for the $28^{\circ}$ case is shown by the dashed line for clear identification. Note that at this $M_{t}$, the fluctuations are the largest for the three intermediate diffusers (see fig. 6). The spectra traces clearly show that most of the energy are at very low frequencies. These frequencies are substantially lower than the 'preferred mode' frequency of the ensuing jet. Possible TollmienSclichting waves in the throat boundary layer or Kelvin-Helmholtz instability in the separated shear layer downstream would also be at much higher frequencies. For a Reynolds number of 215, based on the measured momentum thickness at the throat, the $\mathrm{T}-\mathrm{S}$ frequency can be estimated to correspond to a Strouhal number of about 0.01 , and thus to a nondimensional value, $f D_{t} \sim_{t}=$ 3.7 .

The effect of excitation on the total fluctuation field, for the $20^{\circ}$ diffuser, is shown in fig. 8; the excitation frequency was chosen from preliminary surveys and corresponds to a $f D_{t} v_{t}$ value of 2.2 . The excitation velocity amplitude, measured at the throat on the centerline, was about 2 percent of $U_{t}$. Radial and axial profiles of total $u^{\prime}$ are shown in figs. $8(a)$ 
and (b), respectively. clearly, the large fluctuations are drastically reduced by the excitation inside as well as outside the diffuser. The suppression effect is not characteristic of the probe location and is a global effect. For the case chosen, it is indeed very pronounced and much more than that observed in the backward-facing step as well as that observed in free shear layers. 7

The $u^{\prime}$-spectra measured at the exit plane, with and without excitation, for the $20^{\circ}$ diffuser case are shown in fig. 9. Comparison with the data for the backward-facing step (fig. 5) shows a very similar effect of the excitation, most of the reduction in the energy takes place at the low frequency end. For comparison, note that the momentum thickness at the throat was 0.123 $\mathrm{mm}$ in the diffuser case (fig. 9) and $1.45 \mathrm{~mm}$ in the step case (fig. 5); the Reynolds number based on the momentum thickness was 215 and 660 in the two cases, respectively. The measurement location was about 1240 initial momentum thicknesses away from the throat in fig. 9 and only about 44 initial momentum thicknesses away from the step $(x / H=0.5)$ in fig. 5 .

Referring back to fig. $8(\mathrm{~b})$, it is clear that the unexcited flow involved a relatively large intensity at the throat $\left(x / D_{t}=0\right)$. The $u^{\prime}-$ spectrum there showed similar low frequency energy as in fig. 7 . It is believed that much of the low frequency energy originated in the separated flow downstream but the unsteadiness was felt upstream at the throat. The low frequency energy, as observed in the u'-spectra at the throat centerline, was practically eliminated under the excitation. In the excited case the total $u^{\prime}$ there was almost entirely due to the excitation. Hot-wire surveys showed that a similar reduction of low frequency fluctuations also occurred under the excitation in the throat boundary layer.

The suppression of the fluctuations is accompanied by an increase in the diffuser pressure recovery co-efficient, which is approximated as $c_{p}=-$ $\mathrm{P}_{\mathrm{st}} /\left(\mathrm{P}_{t t}-\mathrm{P}_{\mathrm{st}}\right)$. Here, $\mathrm{P}_{\text {st }}$ and $\mathrm{P}_{t t}$ are the measured static and total pressures at the throat. The $c_{p}$ data with and without excitation are shown in fig. 10. For these data, at each Mach number the excitation frequency as well as available amplitude were roughly scanned to obtain an optimm effect. clearly, for all three diffuser cases shown in fig. 10, the improvement is achieved at low $M_{t}$ where the flow is apparently characterized by the transitional initial boundary layer.

The range of frequency effective in suppressing the fluctuations was determined for three $M_{t}$ values for the $20^{\circ}$ diffuser, and for three diffusers at $M_{t}=0.075$. These data are shown in figs. $11(a)$ and (b). For these data, 
one of the acoustic drivers was used (fig. 2b) and the input voltage to the driver was kept a constant. This resulted in varying amplitude at the throat that depended on the resonance characteristics of the facility. For example, at $M_{t}=0.075$ the amplitudes at $f_{p}=750 \mathrm{~Hz}, 1100 \mathrm{~Hz}$ and $4500 \mathrm{~Hz}$ were about 0.2 percent, 2 percent and 0.15 percent of $U_{t}$, respectively. At a given $f_{p}$ and input voltage, varying $M_{t}$ resulted in an inverse variation in the amplitude. The rigor of keeping the amplitude a constant was avoided due to time constraint and because the excitation effect was found to be relatively insensitive to the amplitude. As long as the right frequency $\left(f_{p}\right)$ was chosen, it required a small amplitude to produce the effect and further increase in amplitude resulted in marginal gains. Thus, the effective excitation frequency ranges should be well represented by the data of fig. 11, even though there is a resonance effect on the data.

For a given diffuser, it is clear that the effect takes place at higher frequencies with increasing $M_{t}$ (fig. 11a). Fig. 11(b) shows that for a given $M_{t}$ the effect occurs approximately in the same frequency range for all three diffusers. Note that fig. 11 (b) indicates a diminishing effect of the excitation with increasing angle of diffusion. Referring back to fig. 6 , one observes that for the larger diffusers (say, $24^{\circ}$ case), at higher $M_{t}$ (say, 0.5) when the throat boundary layer is presumably turbulent, the intensity is relatively higher (about 0.12 ). In comparison, say, for the $16^{\circ}$ case, the intensity is much lower (about 0.006) at the same $M_{t}$. The acoustic excitation is believed to be tripping the the throat boundary layer to a turbulent state. Thus, the excitation may be expected to bring the turbulence intensity down, at the most, to a level that is observed in the mid-Mt range where the boundary layer is turbulent. Presumably, this is why when the excitation is applied at $M_{t}=0.075$ (fig. 11b), the effect is less pronounced for the larger diffusers.

Taking the optimum excitation frequency (fig. 11a) to be $1800 \mathrm{~Hz}$ and $4500 \mathrm{~Hz}$ for $\mathrm{M}_{\mathrm{t}}=0.075$ and 0.15 , respectively, the strouhal number based on the throat velocity and momentum thickness turns out to be about 0.0083 in both cases. This number is somewhat lower than the value 0.017 found to be optimum in free shear layer excitation studies. 7 However, in the diffuser case the separation point could be somewhat downstream of the throat where the core velocity would be lower and momentum thickness higher. This would have an effect of increasing the strouhal number. For the backward-facing step case of fig. 5, the corresponding Strouhal number was about 0.011 . The latter number could be representative of the optimum strouhal number of 
excitation in the wall bounded separated flows. It is somewhat lower than that observed in free shear layer cases, possibly because of the adverse pressure gradient that exists near the separation point in these flows.

\section{Concluding Remarks:}

Acoustic excitation can significantly suppress large amplitude unsteady fluctuations otherwise occurring naturally in wall bounded separated flows. This effect is documented for flows over a backward-facing step and through conical diffusers and compared with similar data for the flow over airfoils.

Let us consider the simpler case of a free shear layer to make the following observation in regards to the mechanism of the suppression effect. As mentioned before, a similar suppression effect, for a variety of free shear layers with nominally laminar initial boundary layers, was reported in ref. 7. It was observed in this earlier work that the initial roll-up frequency of the natural (unexcited) shear layer was always substantially lower (by about 40 percent) than the prediction for the most. amplified disturbance frequency. The latter frequency corresponded to a strouhal number, based on the initial momentum thickness, $s t_{\Theta}=0.017 .11$ Note that the optimum suppression was found to occur when excited at this same $s t_{\theta}$. Why the initial roll up occurred at a frequency lower than the most unstable disturbance frequency of the shear layer had remained unexplained.

An explanation for the lower initial roll up frequency could be the coupling between the Tollmien Schlichting waves occurring upstream of the separation point and the Kelvin-Helmholtz waves occurring downstream. In all cases of ref. 7, as well as the backward facing step and the conical diffuser cases of the oresent study, the Reynolds number based on the initial momentum thickness $\left(R_{\theta}\right)$ fell in the approximate range of 200-700. This is a range where the T-S waves are expected to develop in the boundary layer. The $S t_{\theta}$ range, corresponding to the branch II neutral stability of a zero pressure gradient boundary layer, 12 for the above $R_{\theta}$ range is about $0.007-0.01$. Thus, in the upstream boundary layer the T-S waves are expected to occur in that $S t_{\Theta}$ range. This in turn would excite the $\mathrm{K}-\mathrm{H}$ waves in the separated shear layer explaining the lower value of the $s t_{\theta}$ corresponding to the initial roll up frequency. Note that the momentum thickness before and after separation should not change significantly. 13

A significance of the lower initial strouhal number is that the corresponding disturbances have a tendency to persist farther downstream in 
these flows. There is ample evidence that relatively lower $s t_{\Theta}$ components also grow to much larger amplitudes even though the amplification rates are lower. For example, in the data of ref. 13 it can be seen that a disturbance at $s t_{\Theta}=0.008$ reaches much larger amplitude, about three times larger, than the maximum amplitude attained by a $s t_{\theta}=0.017$ disturbance. Thus, the lower initial strouhal number in the natural shear layers reconciled with the fact that the ensuing flow fluctuations were of large amplitudes.

Therefore, the upstream boundary layer develops the T-S waves prior to the separation which in turn excite the $\mathrm{K}-\mathrm{H}$ waves in the separated shear layer. The separated shear layer is thus in a state of 'self excitation', and furthermore, the 'excited' instability waves are at low strouhal numbers which persist farther downstream. This causes the larger amplitude flow fluctuations downstream. The acoustic excitation at $s t_{\Theta}=0.017$ overrides this scenario and the amplification and 'saturation' of the forced instability wave occurs much faster at a farther upstream location. This results in a flow field that is similar to one having a initially turbulent boundary layer in which the flow fluctuations downstream are of smaller amplitudes compared to the transitional case. In a sense, therefore, the suppression is achieved via a change in the initial condition effected by the acoustic excitation.

It appears that the same explanation of the mechanism should apply to some of the wall bounded separated flow cases also. It is quite plausible that a similar mechanism is at play in the backward facing step as well as the diffuser cases, even though the flows are much more complex due to the presence of the wall. In these cases the values of the excitation strouhal number $\left(s t_{\theta}\right)$ for optimum effect are somewhat lower presumably because of the non-zero pressure gradients near the separation points.

In the airfoil case, the scenario of self excitation through $\mathrm{T}-\mathrm{S}$ waves exciting the $\mathrm{K}-\mathrm{H}$ waves does not seem likely. Even though the boundary layer thickness could not be measured because of the lange unsteady fluctuations, it is reasonable to believe that $R_{\theta}$ prior to separation was very low to preclude the formation of the $T-S$ waves. The mechanism of the resonance like flow oscillation in this case, at unusually low frequencies, 5 have remained unexplained. However, the effect of the acoustic excitation have similarities with the other cases considered. This is why it was conjectured in ref. 5 that a transitional state may be necessary near the separation point in order for the resonance like oscillation to take place. 
Several other questions remain unanswered for the wall bounded separated flows. By far the most important of these is the question regarding the origin of the low frequency unsteady fluctuations. There have been many reports of such unusually low frequency fluctuations in a variety of separated flows. But unfortunately very little is known about the mechanisms. This, in fact, is a focus in our continuing effort in this area and investigations are under way primarily with the backward-facing step as the test case. 14

Finally, it must be emphasized that the low frequency unsteadiness is not only characteristic of initially laminar or transitional boundary layers. There is ample evidence that it also occurs in flows with fully turbulent initial condition. It appears that an initially transitional state substantially augments the low frequency unsteadiness. The present data demonstrate that this can be trimmed down by the acoustic excitation. Whether the unsteady fluctuations for the fully turbulent initial boundary layer case can be reduced, and if so to what extent, also remain unanswered at this time.

\section{References}

1. Smith, C. R. and Layne, J. L., "An experimental investigation of flow unsteadiness generated by transitory stall in plane-wall diffusers", $\mathrm{J}$. Fluids Engineering, vol. 101, pp. 181-185, June 1979.

2. Ashjaee, J. and Johnston, J. P., "Straight-walled, two-dimensional diffusers -- transitory stall and peak pressure recovery", J. of Fluids Engineering, vol. 102, pp. 275-282, sept 1980.

3. Eaton, J. K. and Johnston J. P., "A review of research on subsonic turbulent flow reattachment", AIAA J., 19(9), pp. 1093-1100, 1981.

4. Simpson, R. L., "Two-dimensional turbulent separated flow", AGARDograph 287, 1985.

5. Zaman, K. B. M. Q., MaKinzie, D. J. and Rumsey, C. L. "A natural lowfrequency oscillation of the flow over an airfoil near stalling conditions", J. Fluid Mech., 202, pp. 403-442, 1989.

6. Zaman, K.B.M.Q. and Potapczuk, M.G., "The Low Frequency Oscillation in the Flow over a NACA0012 Airfoil with an 'Iced' Leading Edge", Proc. Low Re Aerodymamics Conference (ed. T.J. Mueller), 1989.

7. Zaman, K.B.M.Q. and Hussain, A.K.M.F., 1981, "Turbulence Suppression in Free Shear Flows by Controlled Excitation", J. Fluid Mech. 103, pp. 133-159.

8. Mechel, F., Mertens, P. and Schilz, W., "Research of sound propagation in sound-absorbent ducts with superimposed air streams", Technical Doc. Rep. No. AMRI-TDR-62-140 (IV), University of Goettingen, 1963. 
9. Zaman, K.B.M.Q. and Dahl M.D., 1990, "Some Observations on Transitory Stall in Conical Diffusers", 28th Aerospace Sciences Meet., Reno, Nevada, AIAA Paper 90-0048.

10. Sohn, K. H., O'brien, J. E. and Reshotko, E., "Some characteristics of bypass transition in a heated boundary layer", Proc. 7th Symp. on Turb. Shear Flows, (also NASA TM-102126), 1989.

11. Michalke, A., "Spatially growing disturbanes in an invisuld : inedl layer", J. Fluid Mech., 23, p. 521, 1965.

12. Saric, W. S. and Nayfeh, A. H., "Nomparallel stability of boundary-layer flows", The Physics of Fluids, 18, pp. 945-950, 1975.

13. Freymuth, P., "On transition in a separated laminar boundary layer", $\mathrm{J}$. Fluid Mech., 25, pp. 683-704, 1966.

14. Zaman, K.B.M.Q. and Rice E. J., "Low frequency oscillation in separated flows", presented at the 43rd Annual Meeting of American Physical Society (DFD), Cornell University, Ithaca, NY, Nov. 18-20, 1990. 


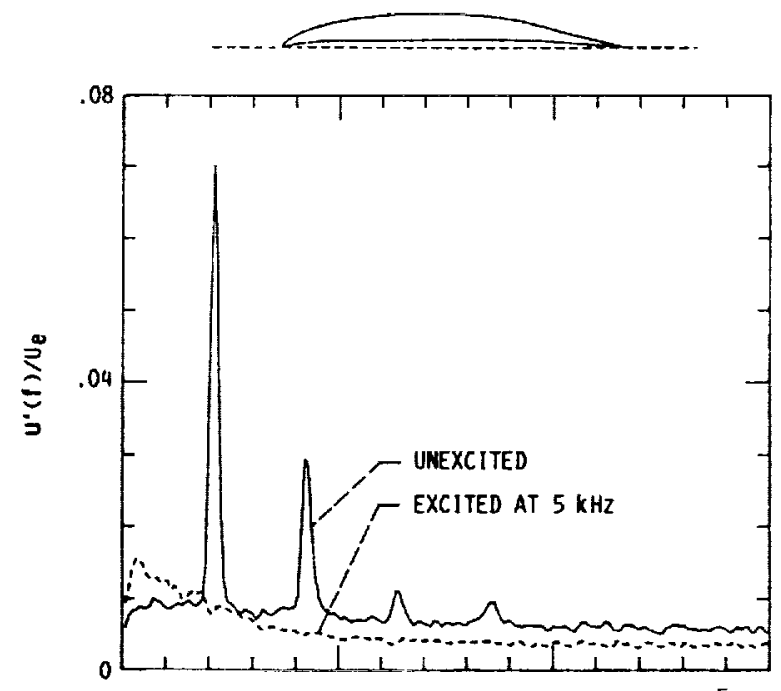

(a) 'LRN' AIRFOIL AT $a=15^{\circ}, R_{c}=0.75 \times 10^{5}$.

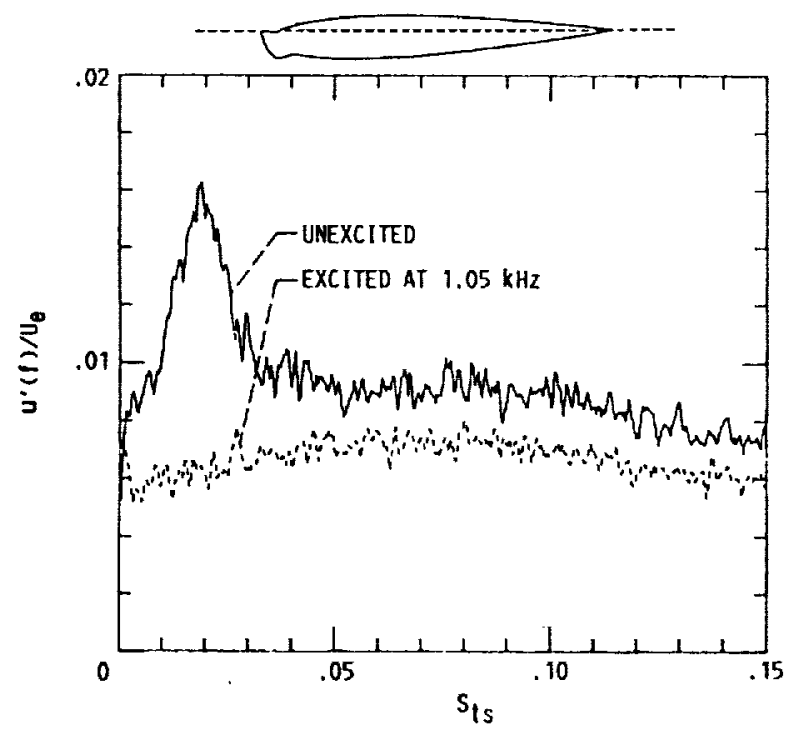

(b) 'ICED' AIRFOIL AT $a=8.5^{\circ}, R_{C}=10^{5}$.

FIGURE 1. - MAKE VELOCITY SPECTRA WITH AMD WITHOUT EXEXCITATION (FRON REF. 6).

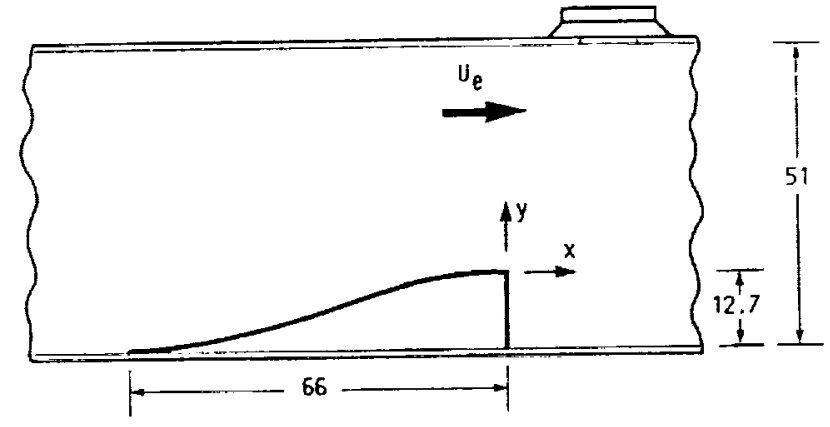

(a) BACKWARD-FACING STEP MODEL IN WIND TUNNEL.

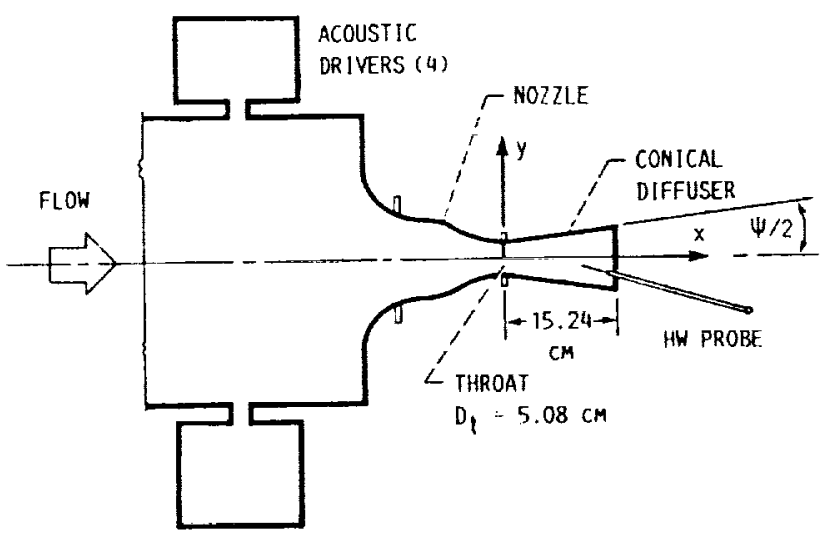

(b) CONICAL DIFFUSER ATTACHED TO AN OPEN JET FACILITY.

FIGURE 2. - SCHEMATIC OF FLOW FACILITIES.

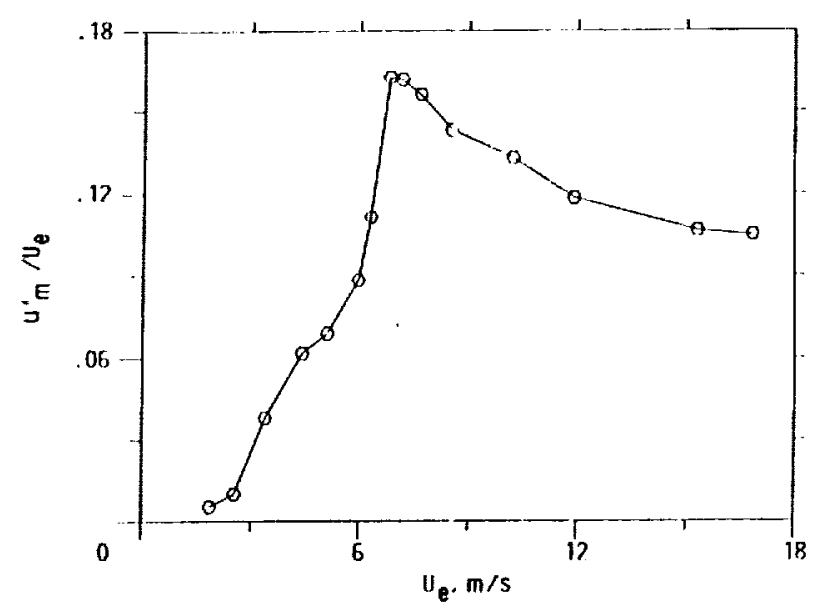

FIGURE 3. - MAXIMUM FLUCTIATION INICNSITY VERSUS FREESTREAM VELOCIIY MEASURED IN THE SEPARAIIMG BOUNDARY LAYER (AT $x=0$ ) IN THE BACKHARD-TACING SIEP. 

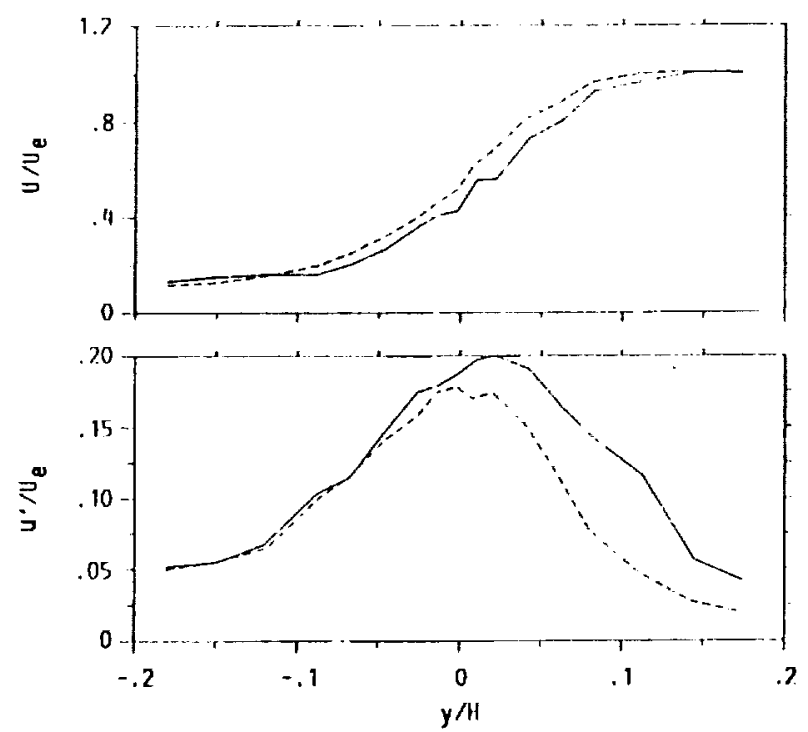

FIGURE 4. - MEAN VELOCITY AND FLUCTUATION INIENSITY PROFILES MEASURED AT $X / H=0.5$ FOR THE BACKWARDFACING SIEP FLON. $U_{0}=6.8 \mathrm{~ms}^{-1}$, SOLID LINE TOR NO EXCITATION, DASHED LIME FOR EXCITAIION AT $50 \mathrm{HZ}$ WITH $u_{e}^{*} / U_{e}=0.2$ PERCENT.

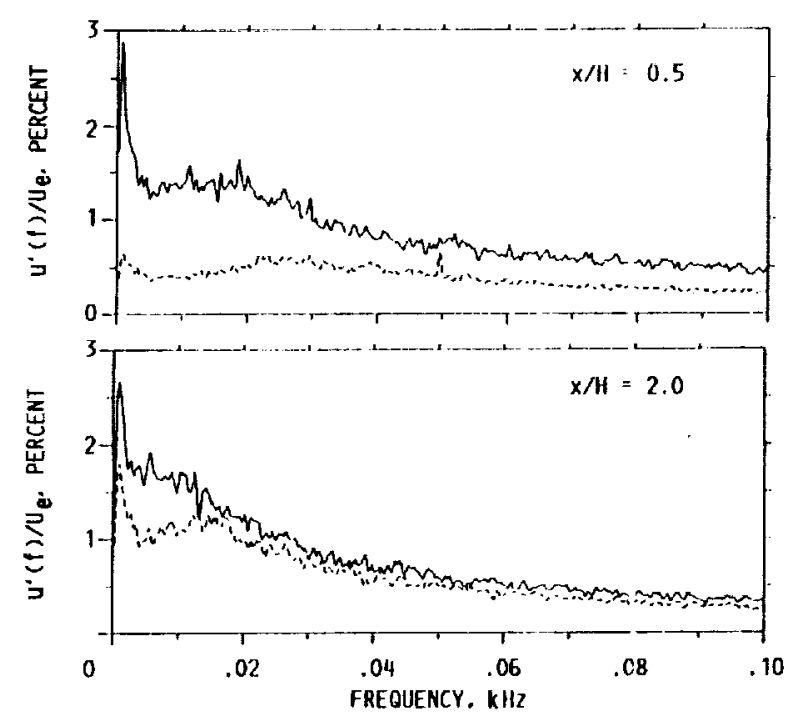

FIGURE 5. - R.M. 5. VELOCITY SPFCTRA MEASURED NIAR TIIE HIGH-SPEED EDGE OF THE MIXING LAYER IN IHE BACKWARDFACING SIEP AT IHDICATED $X / H$. SOL.ID LINE. FOR NO EXCITATION, DASIED LINE TOR EXCITATION AS IN FIG. 4.

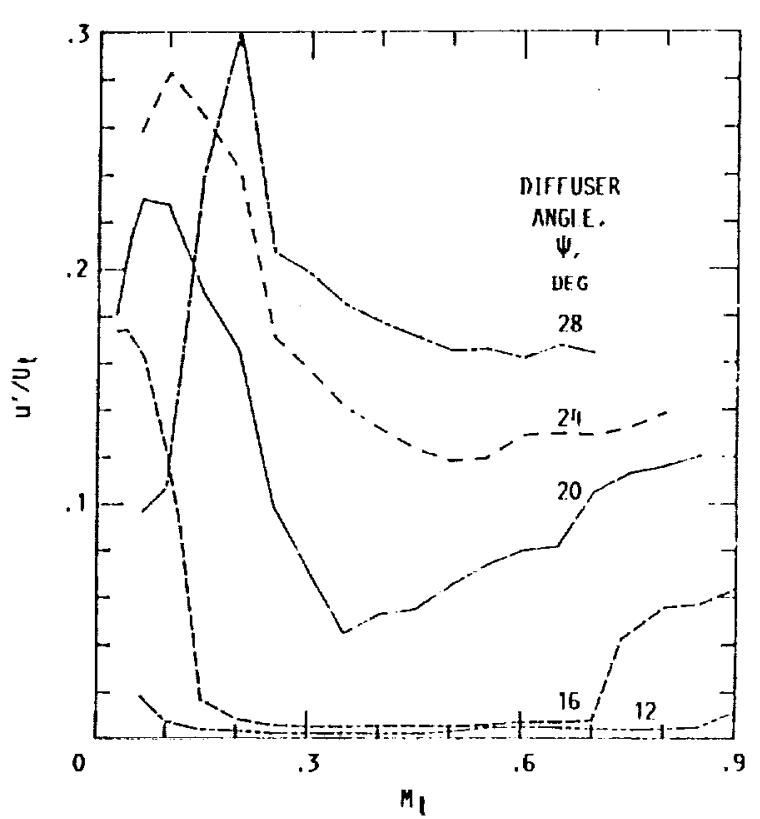

FIGURE 6. - TOTAL R.M.S. TLUCTUATION INIENSITY MIASURED AT THE EXII PLANE OF THE CONICAL DIFFUSERS $\left(x / D_{t}=3, y / D_{t}=0.25\right)$, AS A FUNCTION OF IHRONI MCH NUMBER. Mt.

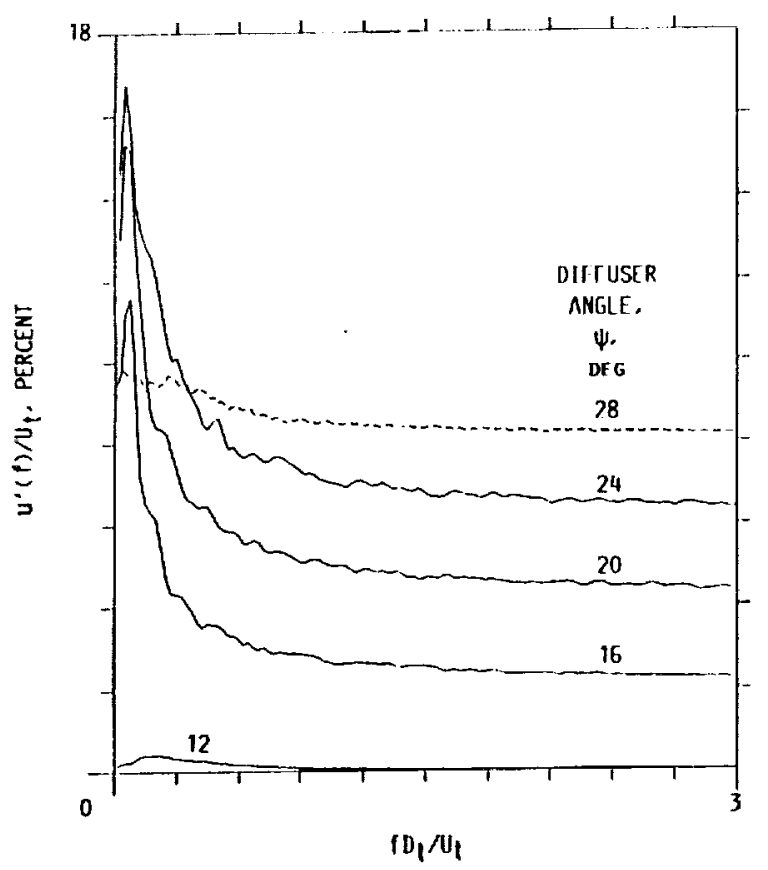

FIGURE 7. - R.M.S. VELOCITY SPECIRA $A T M_{t}=0.075$. FOR IHE FIVE DIFFUSERS: $x / D_{t}=3, y / D_{t}=0.25$. CURVES ARE SIAGGERED BY ONE ORDINAIE DIVISION. 


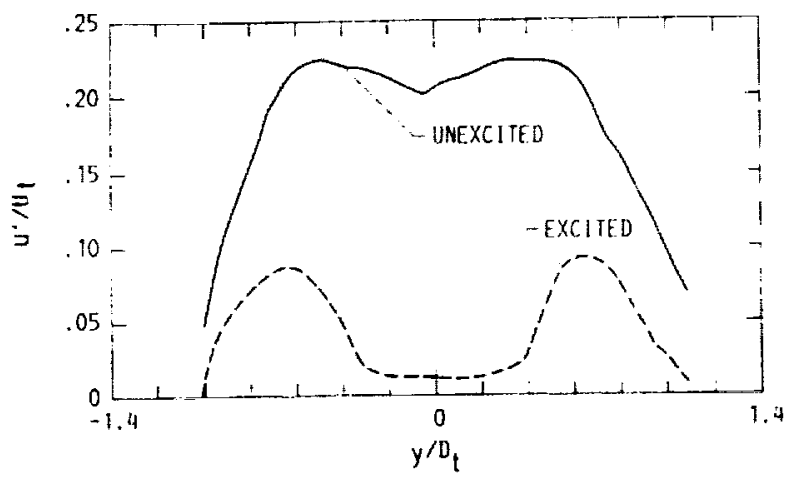

(a) RADIAL PROFILES AT EXIT $\left(x / D_{1}=3.05\right)$.

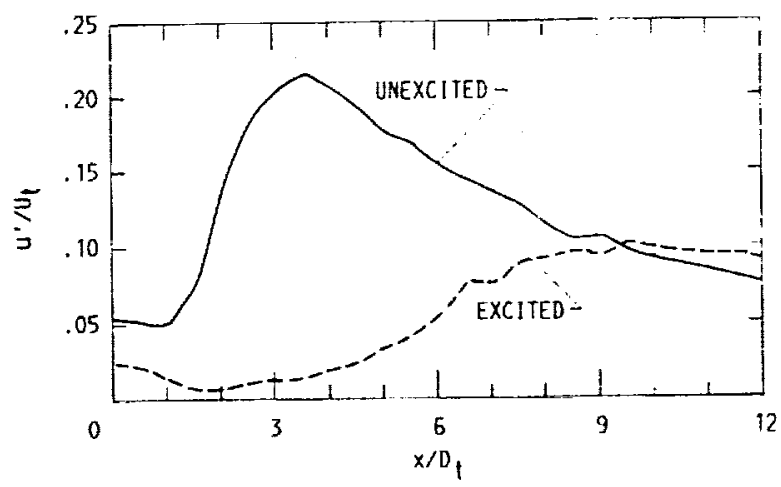

(b) AXIAL PROFILES ALONG CENTERLINE.

F1GURE 8. - PROFILES OF TOTAL R.M.S. FLUCTUATION INTENSITY. WITH AND WITHOUT EXCITATION FOR THE $20^{\circ}$ DIFFUSER: $M_{t}=0.075 . f_{p}=1.1 \mathrm{kHz}$.

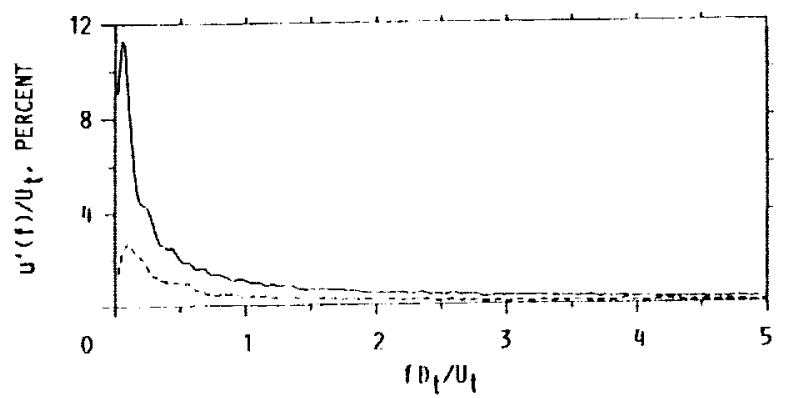

FIGURE 9. - R.M.S. VELOCITY SPECIRA WITH AND WITHOUI EXCITATION $\left(f_{p}=1.1 \mathrm{kHz}\right)$ IOR THE $20^{\circ}$ DITFUSTR AI $M_{1}=0.075 ; x / D_{1}=3 . y / D_{1}=0.25$.

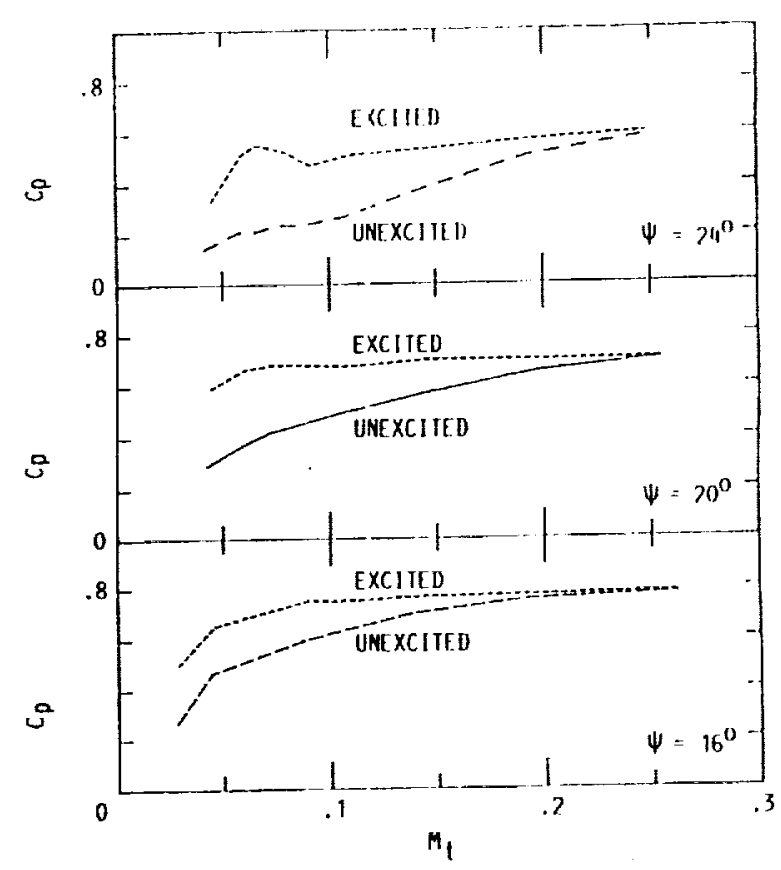

FIGURE 10. - PRESSURE RECOVERY COETF ICITNI VERSUS MA WITH AND WITHOUT ECITAIION FOR THE THREC DIFFUSIRS. 


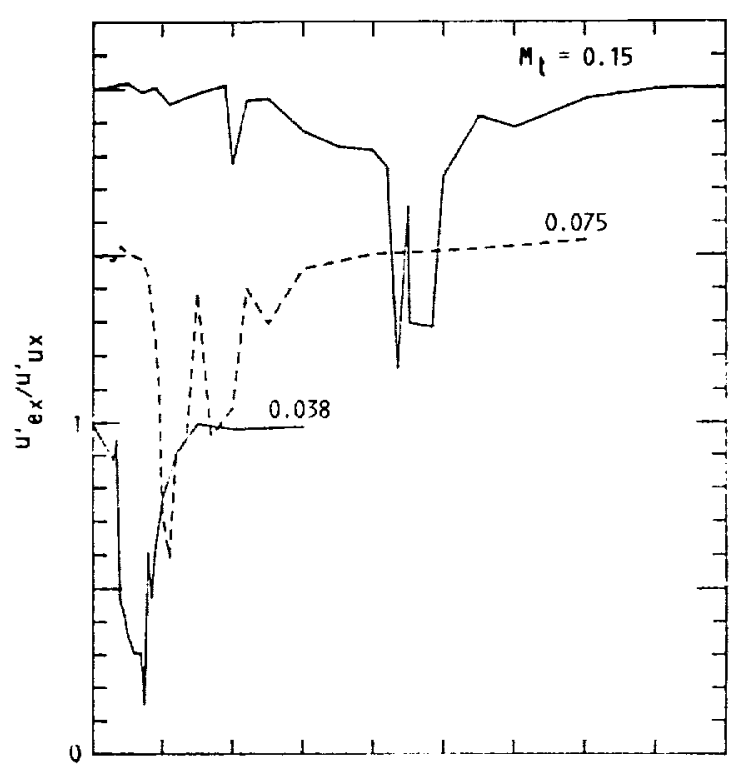

(a) FOR $20^{\circ}$ DIFFUSER AT TIREE $M_{1}$.

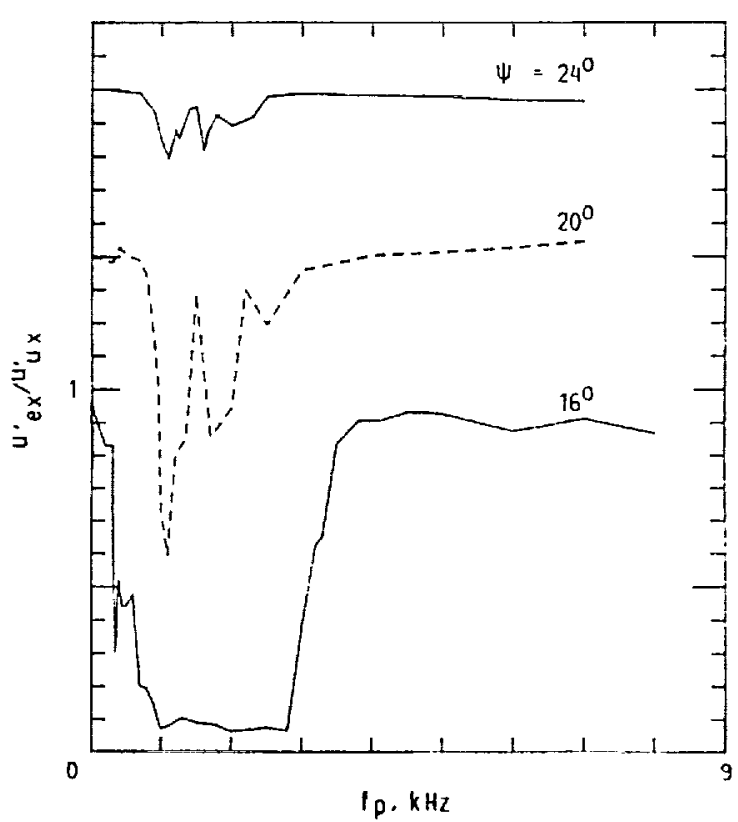

(b) AT $M_{k}=0.075$ FOR THREE DIFFUSERS.

FIGURE 11. - RATIOS OF TOTAL R.M.S. INTENSITIES, WITH AND WITHOUT EXCITATION, AT $x / D_{1}=3$ AND $y / D_{1}=0.25$. CURVES ARE STAGGERED BY ONE MAJOR DIVISION. 


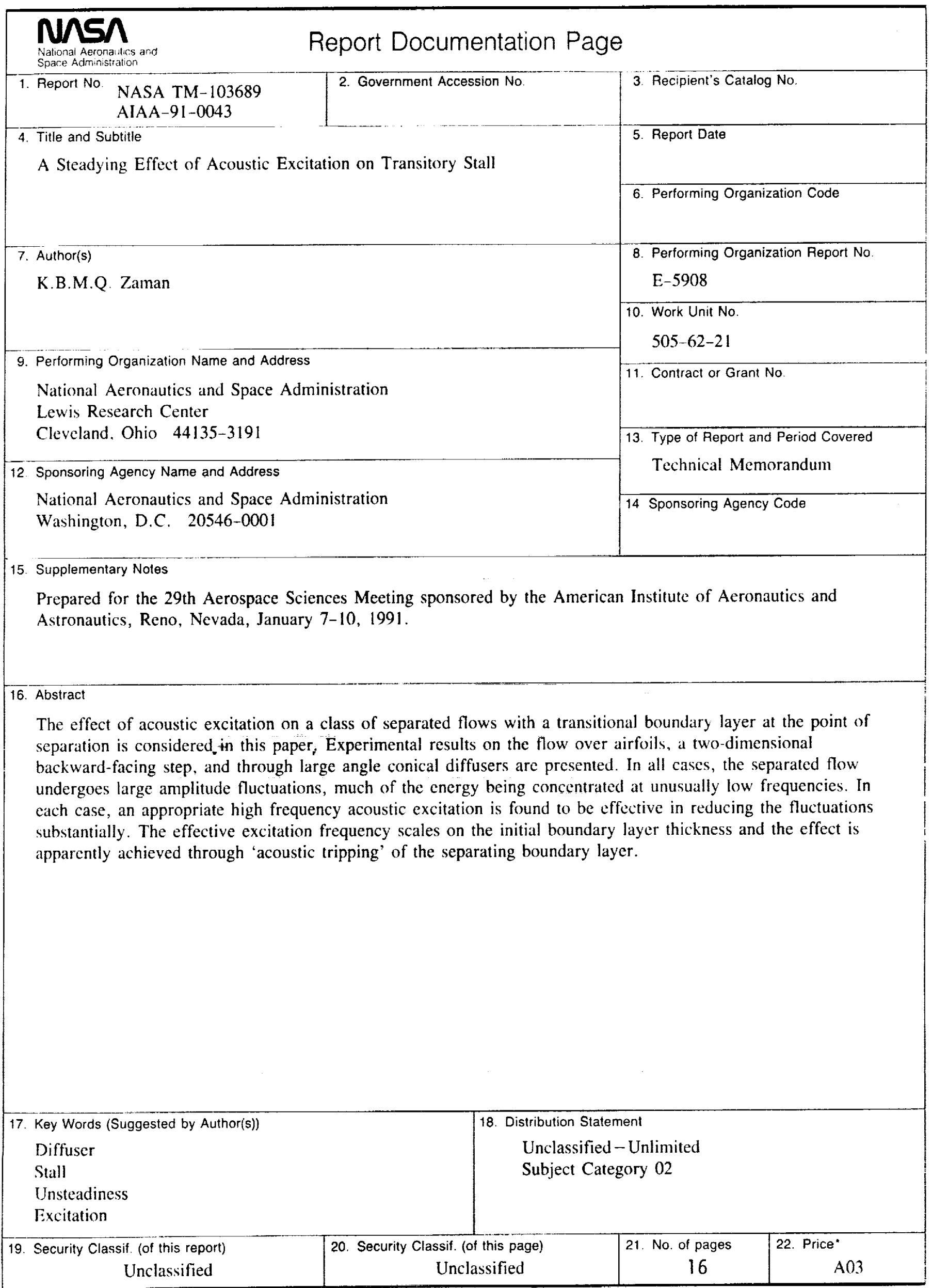

NASA FORM 1626 OCT $86 \quad$ "For sale by the National Technical Information Service Springfield, Virginia 22161 